\title{
A pair of novel targets
}

Fibrosis - the development of excess connective tissue owing to activation of myofibroblasts - is a key process in the pathophysiology of conditions such as lung injury and kidney disease. Two recent papers in PNAS and Nature Medicine highlight potential new targets for these conditions, which are often not responsive to existing drugs.

In the first study, Hecker and colleagues identified NOX4 - the gene that encodes NADPH oxidase 4 (NOX4), which catalyzes the reduction of $\mathrm{O}_{2}$ to reactive oxygen species - as one of the most highly induced genes in human foetal lung mesenchymal cells that had been stimulated with transforming growth factor $\beta 1$ (TGF $\beta 1$ ) to induce differentiation into myofibroblasts. Studies using RNA interference-mediated knockdown of NOX4 in lung mesenchymal cells isolated from individuals with human idiopathic pulmonary fibrosis showed that NOX4 was necessary for TGF $\beta 1$-stimulated hydrogen peroxide production and the induction of $\alpha$-smooth muscle actin (aSMA, a marker of myofibroblast formation) and fibronectin

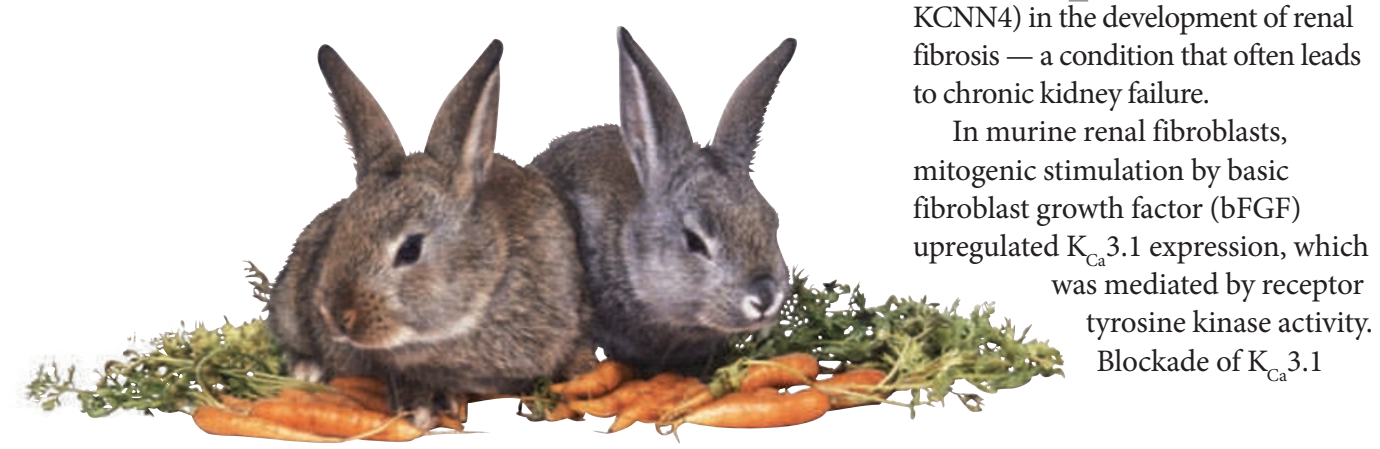

expression. In addition, secretion of soluble collagen by TGF $\beta 1$ stimulated cells, was inhibited by knockdown of NOX4, supporting the involvement of NOX4 in myofibroblast differentiation and proliferation.

Next, the authors studied mouse models of lung injury. NOX4 expression was induced in a timegenic phase of bleomycin-induced lung injury. In this model, and in a hapten-driven lung injury model, delivery of Nox4-specific small interfering RNA mediated an antifibrotic effect. Finally, the flavoenzyme inhibitor diphenyleneiodonium chloride reduced fibrosis in mice that were subjected to bleomycin-induced lung injury, and reduced numbers of aSMA-expressing myofibroblasts in the injured lung.

The second study built on increasing evidence that $\mathrm{Ca}^{2+}$ channels are involved in cellular ular $\mathrm{Ca}^{2+}$ signalling and affecting cell cycle progression. Grgic and colleagues investigated the activity of the intermediate-conductance $\mathrm{Ca}^{2+}$-activated $\mathrm{K}^{+}$channel $\underline{\mathrm{K}}_{\mathrm{Ca}} 3.1$ (also known as KCNN4) in the development of renal fibrosis - a condition that often leads chronic kidney failure.

In murine renal fibroblasts, mitogenic stimulation by basic fibroblast growth factor (bFGF) was mediated by receptor tyrosine kinase activity. Blockade of $\mathrm{K}_{\mathrm{Ca}} 3.1$ dependent manner during the fibroproliferation by enhancing intracell- with the selective inhibitor TRAM-34 reduced proliferation of renal fibroblasts that had been stimulated by bFGF, and caused cell cycle arrest in phase $\mathrm{G}_{0}-\mathrm{G}_{1}$.

In mice that had undergone unilateral ureteral obstruction (UUO) - a model of renal fibrosis - there was more than a 20 -fold increase in $\mathrm{K}_{\mathrm{Ca}} 3.1$ expression in the kidneys, which was accompanied by an increased expression of fibroblastspecific protein 1, collagen I and III and TGF $\beta$. Kidneys from $\mathrm{K}_{\mathrm{Ca}} 3.1$ deficient mice that were subjected to UUO had attenuated chronic tubulointerstitial damage, reduced collagen deposition, fewer aSMA-positive cells and a better preservation of differentiated proximal tubules and total renal parenchyma compared with wild-type mice, showing that progression of renal fibrosis is attenuated by an absence of $\mathrm{K}_{\mathrm{Ca}} 3.1$ channel functions. Finally, in the wild-type UUO model, injections of TRAM-34 attenuated renal fibrosis, which was accompanied by a reduction of chronic tubulointerstitial damage, a decrease in collagen I and III deposition and a significant reduction in interstitial aSMA-expressing cells.

Charlotte Harrison

ORIGINAL RESEARCH PAPERS Hecker, L. et al. NADPH oxidase-4 mediates myofibroblast activation and fibrogenic responses to lung injury. Nature Med. 15, 1077-1081 (2009) (doi:10.1038/nm.2005)|Grgic, I. et al. Renal fibrosis is attenuated by targeted disruption of $\mathrm{K}_{\mathrm{Ca}} 3.1$ potassium channels. Proc. Natl Acad. Sci. USA 106, 14518-14523 (2009) 